\title{
Exploring causality in the association between circulating 25-hydroxyvitamin D and colorectal cancer risk: a large Mendelian randomisation study
}

Yazhou He ${ }^{1,2,3 \dagger}$, Maria Timofeeva ${ }^{1 \dagger}$, Susan M. Farrington ${ }^{1}$, Peter Vaughan-Shaw ${ }^{1}$, Victoria Svinti ${ }^{1}$, Marion Walker ${ }^{1}$, Lina Zgaga ${ }^{1,4}$, Xiangrui Meng ${ }^{3}$, Xue Li $^{3}$, Athina Spiliopoulou ${ }^{3}$, Xia Jiang ${ }^{5,6}$, Elina Hyppönen ${ }^{7,8}$, Peter Kraft ${ }^{5}$, Douglas P. Kiel9,10,11, The SUNLIGHT consortium, Caroline Hayward ${ }^{12}$, Archie Campbell ${ }^{13}$, David Porteous ${ }^{13}$, Katarina Vucic ${ }^{14}$, Iva Kirac ${ }^{15}$, Masa Filipovic ${ }^{16}$, Sarah E. Harris ${ }^{17,18}$, lan J. Deary ${ }^{17,19}$, Richard Houlston ${ }^{20}$, Ian P. Tomlinson ${ }^{21}$, Harry Campbel1 ${ }^{1,3}$, Evropi Theodoratou ${ }^{1,3^{*}}$ and Malcolm G. Dunlop ${ }^{1 *}$ (D)

\begin{abstract}
Background: Whilst observational studies establish that lower plasma 25-hydroxyvitamin D (25-OHD) levels are associated with higher risk of colorectal cancer (CRC), establishing causality has proven challenging. Since vitamin $D$ is modifiable, these observations have substantial clinical and public health implications. Indeed, many health agencies already recommend supplemental vitamin D. Here, we explore causality in a large Mendelian randomisation (MR) study using an improved genetic instrument for circulating 25-OHD.
\end{abstract}

Methods: We developed a weighted genetic score for circulating 25-OHD using six genetic variants that we recently reported to be associated with circulating 25-OHD in a large genome-wide association study (GWAS) meta-analysis. Using this score as instrumental variable in MR analyses, we sought to determine whether circulating 25-OHD is causally linked with CRC risk. We conducted MR analysis using individual-level data from 10,725 CRC cases and 30,794 controls (Scotland, UK Biobank and Croatia). We then applied estimates from meta-analysis of 11 GWAS of CRC risk (18,967 cases; 48,168 controls) in a summary statistics MR approach.

Results: The new genetic score for 25-OHD was strongly associated with measured plasma 25-OHD levels in 2821 healthy Scottish controls $\left(P=1.47 \times 10^{-11}\right)$, improving upon previous genetic instruments (F-statistic 46.0 vs. 13.0). However, individual-level MR revealed no association between 25-OHD score and CRC risk (OR 1.03/unit logtransformed circulating $25-\mathrm{OHD}, 95 \% \mathrm{Cl} 0.51-2.07, P=0.93)$. Similarly, we found no evidence for a causal relationship between 25-OHD and CRC risk using summary statistics MR analysis (OR 0.91, 95\% Cl 0.69-1.19, $P=0.48)$.

\footnotetext{
* Correspondence: e.theodoratou@ed.ac.uk; malcolm.dunlop@ed.ac.uk

${ }^{\dagger}$ Yazhou He and Maria Timofeeva contributed equally to this work.

${ }^{1}$ Colon Cancer Genetics Group, Medical Research Council Human Genetics

Unit, Medical Research Council Institute of Genetics \& Molecular Medicine,

Western General Hospital, The University of Edinburgh, Edinburgh EH4 2XU

UK

Full list of author information is available at the end of the article
}

(c) The Author(s). 2018 Open Access This article is distributed under the terms of the Creative Commons Attribution 4.0 International License (http://creativecommons.org/licenses/by/4.0/) which permits unrestricted use, distribution, and reproduction in any medium, provided you give appropriate credit to the original author(s) and the source, provide a link to the Creative Commons license, and indicate if changes were made. The Creative Commons Public Domain Dedication waiver (http://creativecommons.org/publicdomain/zero/1.0/) applies to the data made available in this article, unless otherwise stated. 
(Continued from previous page)

Conclusions: Despite the scale of this study and employing an improved score capturing more of the genetic contribution to circulating 25-OHD, we found no evidence for a causal relationship between circulating 25-OHD and CRC risk. Although the magnitude of effect for vitamin D suggested by observational studies can confidently be excluded, smaller effects sizes and non-linear relationships remain plausible. Circulating vitamin D may be a CRC biomarker, but a causal effect on CRC risk remains unproven.

Keywords: Vitamin D, Colorectal cancer, Mendelian randomisation

\section{Background}

Colorectal cancer (CRC) is the third most commonly diagnosed cancer worldwide and is one of the leading causes of cancer-specific death [1]. A variety of risk factors have been identified, including low 25-hydroxyvitamin D (25-OHD) [2]. 1,25 dihydroxyvitamin D3 or calcitriol, the active metabolite of 25-OHD, binds to the nuclear vitamin $\mathrm{D}$ receptor and subsequently takes effect by maintaining cellular homeostasis and controlling cell growth $[3,4]$. Postulated mechanisms for the apparent protective effect of 25-OHD include effects on transcriptional regulation of anticancer target genes involved in proliferation, apoptosis, differentiation, inflammation, invasion and metastasis [4]. Meta-analysis of prospective observational studies involving more than one million participants provided evidence of an inverse association between a $10 \mathrm{ng} / \mathrm{mL}$ increment in circulating 25-OHD level and a $26 \%$ decreased $\mathrm{CRC}$ risk $[5,6]$. Given the high prevalence of vitamin D deficiency worldwide [7], especially for high latitude areas such as Scotland [8], and the fact that deficiency can be rectified by dietary supplementation, there is compelling rationale to investigate the contribution of 25-OHD to CRC incidence in the general population.

The associations between vitamin D and CRC reported in observational studies could be biased by reverse causality or confounding factors. Potential confounding factors include body mass index (BMI) [9], diet low in vitamin D, or amount of time spent outdoors [10], each of which may separately influence CRC risk. These could potentially compromise true benefits of any interventions on circulating 25-OHD level. Although the effect of modifying 25-OHD levels can be verified by traditional randomised controlled trials of vitamin D supplementation, these would be prohibitively costly and lengthy in duration. The "VITamin D and OmegA-3 TriaL (VITAL)" was launched in 2010 to investigate the effect of vitamin D supplementation on cancer and cardiovascular disease outcomes [11]. Although 20,000 participants will be recruited to the trial, it could still be underpowered to detect the potential effect on a single type of cancer given the relatively low frequency of CRC occurrence.

Mendelian randomisation (MR) is one of the emerging approaches to strengthen causal inference based on the instrumental variable (IV) method [12]. The conceptual framework of MR is shown in Fig. 1a. A typical MR study uses genetic variants as the IV, assuming that risk alleles for a certain phenotype are randomly allocated during gamete formation [13]. There are some basic assumptions for a valid IV in MR studies [14]. The first is the relevance assumption, which means that instrumental genetic variants should be significantly associated with the exposure; the second assumption requires no association between the IV and confounders of the exposure-outcome relationship. The third is the exclusion restriction assumption, indicating that these variants should affect the outcome solely through the exposure. If the MR assumptions are satisfied then the potential causal effect can be inferred based on the observed IV-exposure and IV-outcome associations. Published MR studies so far have not found support a causal relationship between 25-OHD and CRC [15-17]. Our group previously performed two MR studies to investigate the possible causal effects of plasma 25-OHD on CRC risk. We did not detect a significant effect of 25-OHD on CRC risk using the conventional MR approach [15]. However, analysis of Bayesian predictor scores across various hypotheses prioritised causal models accounting for hidden pleiotropy and confounding over the reverse causality hypothesis [18]. The implemented methodology accounted for confounding by unknown factors and allowed pleiotropic relationships; hence, the results are not dependent on strong and often unrealistic assumptions of the classical MR methods.

It is worth noting that, in all previous MR studies, only four genetic variants (rs2282679, rs12785878, rs6013897, rs10741657) [19] were used to build the instrument. Recently, with the sample size of genome-wide association studies (GWAS) accumulating rapidly, two further genetic loci associated with circulating 25-OHD levels were identified (rs10745742 and rs8018720) [20]. Simulation studies found that incorporating more genetic variants into a single instrument by computing genetic risk scores (GRS) could improve the instrument strength and accuracy of estimation $[21,22]$, highlighting the necessity to re-evaluate the causal effect of 25-OHD on CRC.

Therefore, we designed this MR study to obtain causal estimates of the association between 25-OHD and CRC (Fig. 1b). Six genetic variants associated with 25-OHD level 


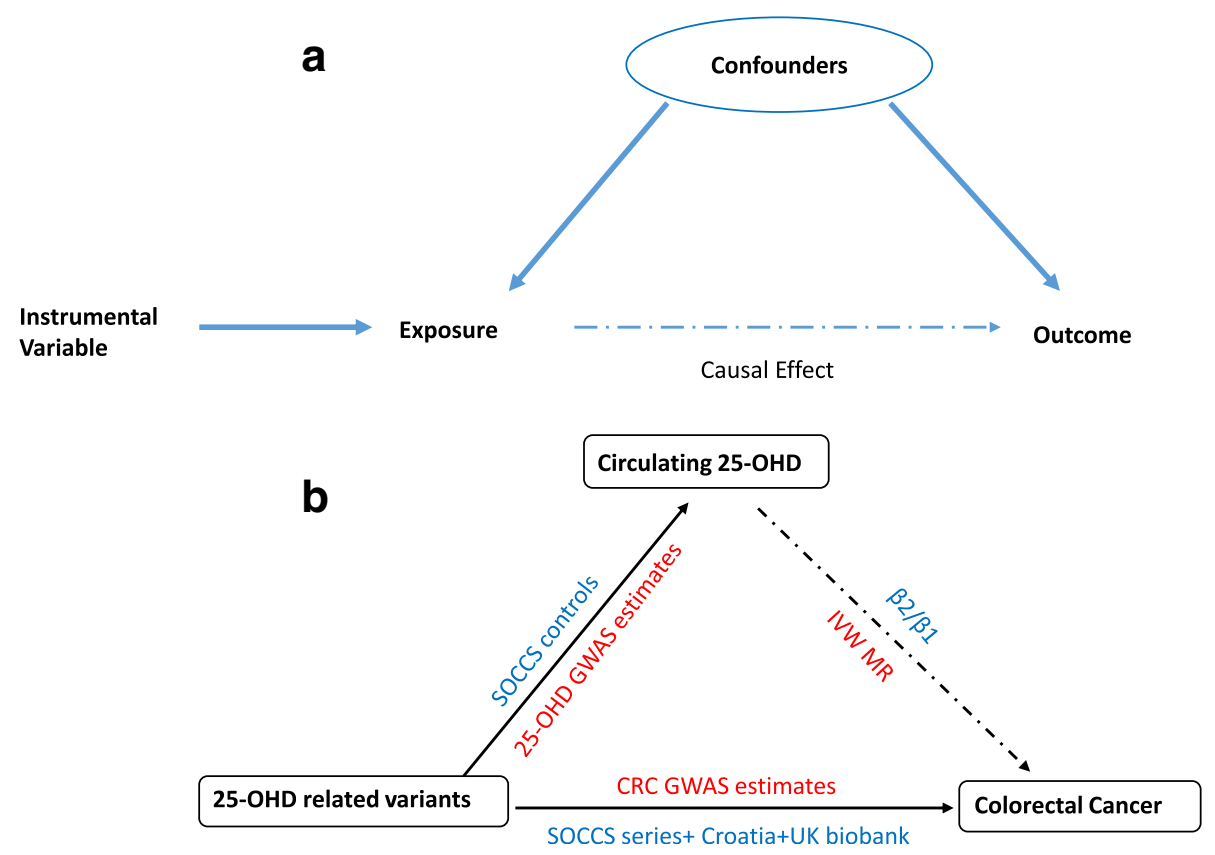

Fig. 1 a Conceptional framework of Mendelian randomisation (MR). The instrumental variable is based on genome-wide significant single nucleotide polymorphisms from independent studies of the association between the exposure of interest (serum 25-hydroxyvitamin D (25-OHD) concentrations) and the outcome (colorectal cancer (CRC)). The effect of an instrumental variable should be independent from the confounding factors and should affect CRC risk only through exposure. In the presence of a causal relationship, the association between instrumental variable and CRC would be expected to be proportionate to its association with the serum 25-OHD concentrations, given the relationship between the serum 25-OHD concentrations and CRC risk. Figure adapted from Timpson et al. [65]. b Basic design of our MR on the causal effect of 25-OHD on CRC risk. The blue text of outer contour showed individual level MR analysis. $\beta 1$ is the regression coefficient of instrumental variable (IV) on exposure (25-OHD level) using controls from the Scotland Colorectal Cancer Study (SOCCS); $\beta 2$ is the regression coefficient of IV on outcome (CRC) using SOCCS series, Croatia and UK biobank case control studies. Causal effect is estimated by the ratio of $\beta 2$ and $\beta 1$. The red text of inner contour showed summary statistics MR analysis. Effect sizes of IVs on 25-OHD and CRC are extracted from two GWAS meta-analyses and causal estimate is derived from an inverse variance-weighted MR analysis

were used as the IV. MR analysis was performed using both individual level data and two-sample summary statistics.

\section{Methods \\ Individual level MR \\ Studies}

Five CRC case-control studies from Scotland, UK and Croatia totalling 10,725 CRC cases and 30,794 controls were included in the individual level MR (Additional file 1: Table S1). The Scottish case-control CRC series consisted of three studies of a total of 6278 cases and 14,692 controls, including (1) 1012 cases and 1012 controls from Scotland 1 (COGS study) [23, 24]; (2) 494 cases from the Study of Colorectal Cancer in Scotland (SOCCS) [25] and 1522 population-based controls without prior history of malignant tumours from the Lothian Birth Cohorts (LBC) 1921 and 1936 [26]; and (3) 4772 cases and 2221 population-based controls from SOCCS [25] and additional 9937 population controls without prior history of CRC from the Generation Scotland-Scottish Family
Health Study (GS:SFHS) [27, 28]. The fourth study included 3683 cases and 15,642 controls matched by age, sex, date of blood draw, ethnicity and region of residence from the UK biobank cohort [29]. Finally, a case-control CRC study from Croatia consisting of 764 cases and 460 population-based controls was also included in the analysis. Details of study genotyping, quality control procedures and imputations are presented in Additional file 1 and elsewhere [30, 31]. A total of 9940 cases and 22,848 controls with genotyping data were included after extensive quality control procedures (Additional file 1). Each study was approved by the respective institutional ethics review board and performed in accordance with the Declaration of Helsinki.

\section{Genetic variants as 25-OHD instruments}

We created an IV for 25-OHD using four genetic variants previously shown to be associated with $25-\mathrm{OHD}$ (rs3755967, rs10741657, rs12785878, rs17216707) [19] and two new single nucleotide polymorphisms (rs10745742, rs8018720) identified by our recent SUNLIGHT Consortium 
GWAS meta-analysis [20]. This meta-analysis of GWAS of serum 25-OHD concentrations included data from SOCCS. To obtain an unbiased IV that could be applied in our study population, a meta-analysis of 29 cohorts including 77,354 individuals of European ancestry was re-run, excluding the SOCCS samples. Summary statistics (including beta estimates for alleles increasing circulating 25-OHD level, standard error and $P$ value) of the genetic variants on 25-OHD were extracted afterwards.

\section{Statistical analysis}

We created a weighted GRS for each individual in SOCCS/GS, UK biobank and Croatia datasets using the six 25-OHD-associated candidate variants. These variants were weighted by effect sizes of 25-OHD increasing alleles from the SUNLIGHT GWAS meta-analysis excluding SOCCS samples. Unweighted GRS was also generated based on the counts of alleles associated with increased level of 25-OHD for each participant.

First, we tested the association between the 25-OHD GRS and log-transformed 25-OHD levels $(\mathrm{nmol} / \mathrm{L})$ in a sub-set of SOCCS controls $(n=2821)$ by applying a univariable linear regression model. We also calculated the F-statistic to evaluate the strength of the genetic instrument, and an F-statistic < 10 was considered as a weak instrument effect [21]. Second, we examined the association between our instrumental GRS of 25-OHD and common confounders including age, sex, BMI, physical activity, assessment centre, smoking status and alcohol consumption based on available data in SOCCS $(n=9746)$ and UK biobank $(n=11,382)$ controls to test the potential violation of the second MR assumption. We also searched the NHGRI-EBI GWAS Catalogue (https://www.ebi.ac.uk/gwas/ accessed in February 2018) to identify any reported associations between the six variants and potential confounders. If the second MR assumption was violated in one of the studies, we performed sensitivity analysis by excluding the corresponding study. We also applied multivariable linear regression models adjusting for age, sex and BMI to obtain the IV-exposure association estimates based on availability of each dataset. Next, the association between GRS and CRC risk was assessed by a logistic regression model in the three Scottish case-control series (Scotland1, SOCCS/ GS, SOCCS/LBC), Croatia and UK biobank datasets, adjusting for age, sex and BMI (based on data availability). Using the coefficient ratio method proposed by Wald [32], we measured the causal effect by calculating the ratio of the IV regression coefficient from the IVoutcome association analysis and the IV regression coefficient from the IV-exposure association, and then estimated the standard error based on the Taylor expansion [33, 34].
Estimates from these five datasets were combined by using the inverse variance meta-analysis under a random effects model. The observed $P$ value $<0.10$ for the $\chi^{2} \mathrm{Q}$ test indicated no significant heterogeneity among included datasets. Considering potential diverse aetiology of tumours in different anatomical locations, we also performed stratified MR analyses in patients with tumours in proximal, distal colon and rectum using available individual-level data.

\section{Summary statistics MR \\ Studies}

We investigated the relationship between the IV for 25-OHD and CRC using summary data from six previously reported GWAS of CRC [30, 31]. Briefly, these GWAS included individuals of European ancestry from the following studies: CCFR1, CCFR2, COIN, FINLAND, UK1 and VQ58 [35-37] (details in Additional file 1: Table S1). Together with the Scottish case-control series, Croatia and UK Biobank studies we included 18,967 cases and 48,168 controls across 11 individual GWASs (Additional file 1: Table S1). Comprehensive details on the cases and controls are available in previously published work [30, 31, 35-37]. After standard quality control procedures, 17,716 cases and 40,095 control individuals were included in the analysis. All studies were approved by their respective institutional review boards and conducted with appropriate ethical criteria in each country and in accordance with the Declaration of Helsinki.

\section{Statistical analysis}

Effects of the six genetic variants on 25-OHD (25-OHD increasing alleles) were extracted from the SUNLIGHT GWAS meta-analysis and effects of these variants on CRC risk were extracted from the CRC GWAS meta-analysis results of 11 case-control studies (Additional file 1: Table S1, Table S6). We also checked if any of the known CRC risk variants were in linkage disequilibrium $\left(\mathrm{r}^{2}>0.01\right)$ with the 25-OHD associated variants in the CRC GWAS meta-analysis results. We applied a range of MR methods using summary genetics data, namely an inverse variance-weighted (IVW) average of associations for IVs [38], and a median-based method [39]. Egger MR [40] was conducted to explore the potential bias introduced by pleiotropy.

IVW MR combines causal effects of candidate variants estimated following the IVW method as proposed by Burgess et al. [38]. As shown by the formula below, $X_{k}$ refers to the effect size of variant $k$ on the exposure, $Y_{k}$ represents the effect size of the same variant on the outcome, and $\sigma_{Y k}$ is the standard error of $Y_{k}$. In addition, to evaluate potential heterogeneity among causal effects of different variants, the $\chi^{2} \mathrm{Q}$ test was 
employed, and a $P$ value of less than 0.10 was regarded as significant heterogeneity.

$$
\begin{aligned}
& \hat{\beta}=\frac{\sum_{k=1}^{K} X_{k} Y_{k} \sigma_{y k}^{-2}}{\sum_{k=1}^{K} X_{k}^{2} \sigma_{y k}^{-2}} \\
& \operatorname{Se}(\hat{\beta})=\sqrt{\frac{1}{\sum_{k=1}^{K} X_{k}^{2} \sigma_{y k}^{-2}}}
\end{aligned}
$$

Considering that unmeasured pleiotropy could lead to violation of the exclusion restriction assumption and bias the MR findings, we employed the MR-Egger regression method that aims to identify and adjust for unbalanced pleiotropy. Additionally, the MR-Egger approach can provide unbiased and minimally biased estimates even in the presence of no causal association and substantial directional pleiotropy [40]. A significant difference of an intercept from zero $(P<0.05)$ suggests existence of unbalanced pleiotropy.

To further evaluate the robustness of possible causal effect when some of the genetic variants in the analysis are not valid IVs and IV assumptions are violated, we also employed median-based methods to derive the causal estimates [39]. As a sensitivity analysis, causal estimates from IVW and MR-Egger were calculated using robust regression in addition to standard linear regression, and penalization of weights of each variant was also applied for IVW, MR-Egger and median-based estimates [41]. A $P$ value of less than 0.05 was considered as statistically significant for causal estimates for our MR. In addition, given these six variants are located in multiple genes with diverse function, which could introduce potential pleiotropy, we also conducted a sensitivity analysis with different combinations of variants, starting with rs10741657 plus rs12785878 (in CYP2R1 and DHCR7 genes affecting 25-OHD synthesis) and sequentially adding rs17216707, rs10745742, rs8018720 and rs3755967.

\section{Power estimation}

We estimated the power of our study according to the method provided by Brion et al. [42]. The six 25-OHD-related variants explained approximately $2.84 \%$ of $25-\mathrm{OHD}$ variation [20]. We fixed the type I error as $\alpha<0.05$ and employed a range of effect estimates from odds ratio (OR) 0.6 to 0.98 per standard deviation increased 25-OHD level. Assuming true causal effect of vitamin D is similar to the effect observed in the SOCCS study (OR 0.83 per standard deviation of increased circulating 25-OHD) we would have a power 0.72 for the individual level approach using 9940 CRC cases and 22,848 controls from the UK biobank, Croatia and Scottish CRC case-control series. The study had sufficient power (80\%) to detect the causal effects of a $19 \%$ or larger decrease in CRC risk per standard deviation increase of 25-OHD. The power for the summary level approach reached 0.80 for a causal effect larger than $14.3 \%$ decreased CRC risk per standard deviation increase of 25-OHD. Power estimation for a range of causal effects as well as proportions of 25-OHD variation explained by the six genetic variants is summarised in Additional file 1: Table S3.

All statistical analyses were performed using PLINK 1.90 and R (version 3.3.0) package 'MendelianRandomization' [43].

\section{Results}

We tested the MR assumptions using SOCCS and UK biobank individual level data. The MR relevance assumption was tested in SOCCS controls $(n=2821)$ with available circulating 25-OHD levels. Both weighted and unweighted GRS were significantly associated with the log-transformed 25-OHD levels in a univariable linear regression model (weighted GRS: $P=1.47 \times 10^{-11}$, unweighted GRS: $\left.P=8.47 \times 10^{-9}\right)$ and after adjustment for age, sex and BMI (weighted GRS: $P=1.37 \times 10^{-11}$, unweighted GRS: $\left.P=5.72 \times 10^{-10}\right)$. We calculated the F-statistic to evaluate the strength of the genetic instrument [21]. The linear regression showed an F-statistic of 46.0 for weighted GRS and 33.7 for unweighted GRS, suggesting the absence of a weak instrument effect $(\mathrm{F}>10)$. The association between the instrument and possible confounders was tested in SOCCS and UK biobank controls. The genetic instrument of six variants on 25-OHD was not significantly associated with any of the common cofounders including age, sex, height, weight, BMI, physical activity, smoking status, alcohol consumption and assessment centre $(P>0.05$, Additional file 1 : Table S2). By searching the GWAS catalogue, we identified no significant association between any of the six variants and common confounders either. None of the known CRC variants were in linkage disequilibrium $\left(r^{2}>0.01\right)$ with the six 25-OHD variants.

No direct association was observed between the weighted or unweighted GRS and CRC risk in SOCCS, Croatia or UK biobank datasets (Table 1). Detailed results of individual level MR analysis for each dataset are summarised in Table 2. Both univariable and multivariable models adjusted for age, sex and BMI, when appropriate, showed no causal effects of 25-OHD on CRC risk in Scotland 1, SOCCS/GS, SOCCS/LBC, Croatia and UK biobank case-control studies. Overall, the result of individual level MR analysis under a multivariable model suggested no significant causal effect of 25-OHD concentration on CRC risk using the weighted GRS (OR 1.03 per unit increased log-transformed 25-OHD, $95 \%$ C $0.51-2.07, P=0.931$ ). No significant heterogeneity 
Table 1 Two-stage regression coefficients for Mendelian randomisation analysis using genetic risk score in individual level data

\begin{tabular}{|c|c|c|c|c|c|c|}
\hline & \multirow{2}{*}{$\begin{array}{l}\text { GRS- } 25 \mathrm{OHD} \text { coefficient }(95 \% \mathrm{Cl})^{\mathrm{b}} \\
\text { sOCCS controls }\end{array}$} & \multicolumn{5}{|c|}{ GRS-CRC coefficient $(95 \% \mathrm{CI})^{c}$} \\
\hline & & Scotland 1 & SOCCS/GS & Croatia & SOCCS/LBC & UK biobank \\
\hline \multicolumn{7}{|c|}{ Univariable model } \\
\hline $\begin{array}{l}\text { Weighted } \\
\text { score }\end{array}$ & 0.055 (0.039 to 0.071$)$ & $\begin{array}{l}0.036 \\
(-0.055 \text { to } 0.128)\end{array}$ & $\begin{array}{l}-0.009 \\
(-0.045 \text { to } 0.026)\end{array}$ & $\begin{array}{l}0.072 \\
(-0.048 \text { to } 0.191)\end{array}$ & $\begin{array}{l}-0.029 \\
(-0.133 \text { to } 0.076)\end{array}$ & $\begin{array}{l}-0.022 \\
(-0.061 \text { to } 0.017)\end{array}$ \\
\hline $\begin{array}{l}\text { Unweighted } \\
\text { score }\end{array}$ & 0.046 (0.031 to 0.062$)$ & $\begin{array}{l}0.037 \\
(-0.055 \text { to } 0.128)\end{array}$ & $\begin{array}{l}-0.005 \\
(-0.041 \text { to } 0.030)\end{array}$ & $\begin{array}{l}0.110 \\
(-0.010 \text { to } 0.230)\end{array}$ & $\begin{array}{l}0.001 \\
(-0.104 \text { to } 0.106)\end{array}$ & $\begin{array}{l}-0.008 \\
(-0.047 \text { to } 0.031)\end{array}$ \\
\hline \multicolumn{7}{|c|}{ Multivariable model ${ }^{a}$} \\
\hline $\begin{array}{l}\text { Weighted } \\
\text { score }\end{array}$ & 0.057 (0.040 to 0.073 ) & $\begin{array}{l}0.109 \\
(-0.011 \text { to } 0.230)\end{array}$ & $\begin{array}{l}-0.026 \\
(-0.081 \text { to } 0.029)\end{array}$ & $\begin{array}{l}0.074 \\
(-0.047 \text { to } 0.194)\end{array}$ & $\begin{array}{l}-0.031 \\
(-0.136 \text { to } 0.074)\end{array}$ & $\begin{array}{l}-0.024 \\
(-0.047 \text { to } 0.031)\end{array}$ \\
\hline $\begin{array}{l}\text { Unweighted } \\
\text { score }\end{array}$ & 0.051 (0035 to 0.068 ) & $\begin{array}{l}0.080 \\
(-0.038 \text { to } 0.197)\end{array}$ & $\begin{array}{l}-0.022 \\
(-0.077 \text { to } 0.032)\end{array}$ & $\begin{array}{l}0.114 \\
(-0.007 \text { to } 0.235)\end{array}$ & $\begin{array}{l}-0.003 \\
(-0.108 \text { to } 0.102)\end{array}$ & $\begin{array}{l}-0.011 \\
(-0.050 \text { to } 0.029)\end{array}$ \\
\hline
\end{tabular}

${ }^{a}$ Multivariable regression model adjusted by age, sex and BMI for Scotland 1, SOCCS/GS and UK biobank, age and sex for Croatia, sex for LBC.MD

${ }^{\mathrm{b}}$ Change in log-transformed 25-OHD (nmol/L) per unit increase in GRS

'Change in logit CRC risk per unit increase in GRS

25-OHD 25-hydroxyvitamin D, Cl confidence interval, CRC colorectal cancer, GRS genetic risk score, LBC Lothian Birth Cohort, SOCCS Scotland Colorectal Cancer Study

was observed among each dataset $\left(P_{\text {het }}=0.227\right)$. Similarly, we did not find a statistically significant causal effect when an unweighted GRS was employed as the IV (OR 1.12, $95 \%$ CI $0.51-2.45, P=0.785)$. The results of stratified analysis did not support a significant causal effect of 25-OHD on risk for proximal, distal or rectal tumours (detailed results in Additional file 1: Table S5).

As shown in Fig. 2, for the summary statistics IVW MR, no statistically significant causal effect of 25-OHD on CRC risk was identified either (OR 0.91 per unit increased log-transformed 25-OHD, 95\% CI 0.69-1.19, $P=0.475)$. MR-Egger regression did not identify evidence of significant horizontal pleiotropy $(P=0.657)$ and the MR-Egger analysis did not observe any statistically significant causal effect (OR 0.83, 95\% CI 0.51-1.34, $P=0.452$ ). In addition, no significant heterogeneity was detected among the causal estimates of the six variants $\left(P_{\text {het }}=0.547\right)$. Effects of each single variant on both 25-OHD and CRC are presented in Table 3. Estimates derived from the median-based methods did not show a statistically significant causal effect (simple median method: OR 0.80, 95\% CI 0.49-1.30, $P=0.375$ ). Detailed results using standard linear regression, robust regression and penalisation are summarised in Table 4. Sensitivity analysis using different combinations of variants did not identify any significant causal effects either (detailed results presented in Additional file 1: Table S4).

\section{Discussion}

In the largest MR study to date, we employed a new IV comprising a genetic score that captures more of the genetic contribution to circulating 25-OHD than has ever been possible before, linked to a large meta-analysis of GWAS for CRC risk in well-matched European populations with similar ambient exposure to vitamin D-making UVB sunlight. We aimed to determine whether the relationship between 25-OHD and CRC risk was causal. We employed several MR methods, including individual level MR analysis, summary level IVW, Egger MR and median-based MR. We used six genetic

Table 2 Main results of Mendelian randomisation analysis using individual level data

\begin{tabular}{|c|c|c|c|c|c|c|c|c|}
\hline \multirow[t]{3}{*}{ Cases/controls } & \multicolumn{5}{|c|}{ Causal estimate (odds ratio) ${ }^{\mathrm{b}}(95 \% \mathrm{Cl})$} & \multirow{3}{*}{$\begin{array}{l}\text { Overall } \\
\text { estimate }^{c}\end{array}$} & \multirow[t]{3}{*}{$P$ value } & \multirow[t]{3}{*}{$P_{\text {het }}^{d}$} \\
\hline & Scotland 1 & SOCCS/GS & Croatia & SOCCS/LBC & UK biobank & & & \\
\hline & 932/942 & $4551 / 8804$ & $689 / 441$ & $461 / 1444$ & $3301 / 11382$ & & & \\
\hline \multicolumn{9}{|l|}{ Univariable model } \\
\hline Weighted score & $1.92(0.36-10.27)$ & $0.84(0.44-1.62)$ & $3.69(0.41-33.34)$ & $0.59(0.09-3.99)$ & $0.67(0.33-1.38)$ & $0.85(0.55-1.33)$ & 0.481 & 0.531 \\
\hline Unweighted score & $2.20(0.30-16.19)$ & $0.89(0.41-1.93)$ & $10.71(0.71-161.41)$ & $1.02(0.11-9.84)$ & $0.85(0.36-1.96)$ & $1.03(0.61-1.73)$ & 0.920 & 0.440 \\
\hline \multicolumn{9}{|l|}{ Multivariable model $^{\mathrm{a}}$} \\
\hline Weighted score & $6.85(0.77-60.81)$ & $0.63(0.24-1.67)$ & $3.82(0.41-35.23)$ & $0.57(0.08-3.87)$ & $0.87(0.44-1.73)$ & $1.03(0.51-2.07)$ & 0.931 & 0.227 \\
\hline Unweighted score & $4.70(0.46-48.41)$ & $0.65(0.22-1.88)$ & $11.21(0.76-164.33)$ & $0.93(0.10-8.66)$ & $0.81(0.38-1.75)$ & $1.12(0.51-2.45)$ & 0.785 & 0.222 \\
\hline
\end{tabular}




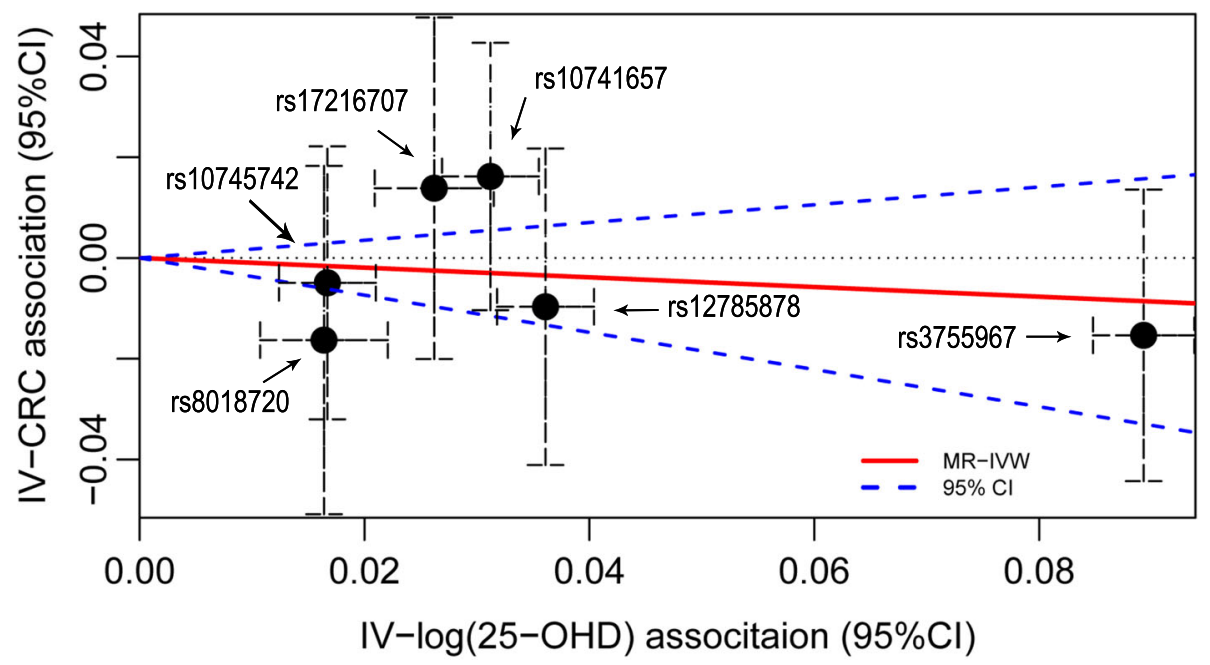

Fig. 2 Association of 25-hydroxyvitamin D (25-OHD) affecting genetic variants with log transformed 25-OHD concentration and colorectal cancer risk. The slope of the red line is the causal estimate derived from inverse variance-weighted (IVW) Mendelian randomisation and slope of the blue dash line represents the $95 \%$ confidence interval of IVW estimate

variants (rs3755967, rs12785878, rs17216707, rs10741657, rs10745742, rs8018720) associated with 25-OHD serum levels as IVs [20]. However, none of the implemented approaches supported a causal association between lower plasma 25-OHD and elevated CRC risk.

Previous retrospective and prospective observational studies establish beyond all reasonable doubt that there is an association between lower circulating 25-OHD levels and elevated CRC risk $[5,6]$. The issue is whether this is a causal relationship. However, randomised controlled trials have failed to demonstrate beneficial effects of vitamin D supplementation on CRC or colorectal adenoma recurrence as an intermediate endpoint. For instance, the Women's Health Initiative trial did not show any effects of $1000 \mathrm{mg}$ of elemental calcium and $400 \mathrm{IU}$ of vitamin $\mathrm{D}_{3}$ supplementation on CRC incidence among postmenopausal women [44]. Similarly, daily supplementation with vitamin $\mathrm{D}_{3}$ (1000 IU), calcium $(1200 \mathrm{mg}$ ) or both after removal of colorectal adenomas did not reduce the risk of recurrent colorectal adenomas [45]. Albeit questioning the potential causal role of 25-OHD in the development of $\mathrm{CRC}$, these trials are widely criticised for short follow-up or lacking proof for effective 25-OHD modification (due to low dose of supplementation) [46-48]. More recently, in human studies, it has been shown that functional genetic variants in the vitamin $\mathrm{D}$ receptor may also influence any protective response to vitamin $\mathrm{D}$ in preventing adenomas, which merits further stratified investigation of the possible effect [49]. Similarly, experimental studies using rodent models of colon cancer treated with high dietary vitamin D were inconsistent in their conclusions. In particular, a causal relationship between high dietary vitamin $\mathrm{D}$ and low colon cancer risk was supported by studies using a mouse model of bacteria-driven colitis and colon cancer [50], and in mice fed with new Western-style diet [51], but not in a rat model of familiar colon cancer [52].

A randomised trial in average risk populations of sufficient size and duration to establish definitively whether or not vitamin D supplementation prevents CRC as the primary endpoint seems unlikely to ever be feasible. Hence, MR methods offer an alternative approach that might provide clarity on whether 25-OHD is causally associated with CRC risk. There is a pressing need for

Table 3 Summary of genetic variants used as instrumental variables in summary statistics approach

\begin{tabular}{llllllll}
\hline ID & Gene & Effect allele & Chromosome & Beta (25-OHD) & Se (25-OHD) & Beta (CRC) & Se (CRC) \\
\hline rs10741657 & CYP2R1 & A & 11 & 0.0312 & 0.0022 & 0.0162 & 0.0135 \\
rs10745742 & AMDHD1 & T & 12 & 0.0167 & 0.0022 & -0.0049 & 0.0138 \\
rs12785878 & NADSYN1/ DHCR7 & $T$ & 11 & 0.0361 & 0.0022 & -0.0097 & 0.016 \\
rs17216707 & CYP24A1 & T & 20 & 0.0262 & 0.0027 & 0.0138 & 0.0173 \\
rs3755967 & GC & C & 4 & 0.0893 & 0.0023 & -0.0154 & 0.0148 \\
rs8018720 & SEC23A & $G$ & 14 & 0.0164 & 0.0029 & -0.0163 & 0.0176 \\
\hline
\end{tabular}

Beta regression coefficients of GWAS meta-analysis, se standard error, CRC colorectal cancer 
Table 4 Main results of Mendelian randomisation analysis in 18,967 colorectal cancer cases and 48,168 controls using summary statistic approach

\begin{tabular}{llll}
\hline Methods & Causal estimates $(95 \% \mathrm{Cl})^{\mathrm{a}}$ & $P$ value & $P_{\text {int }}$ \\
\hline IVW & $0.91(0.69-1.19)$ & 0.475 & $\mathrm{NA}$ \\
Robust IVW & $0.90(0.74-1.09)$ & 0.318 & $\mathrm{NA}$ \\
Penalised IVW & $0.91(0.69-1.19)$ & 0.475 & $\mathrm{NA}$ \\
MR-Egger & $0.83(0.51-1.34)$ & 0.452 & 0.657 \\
Robust MR-Egger & $0.83(0.67-1.03)$ & 0.09 & 0.61 \\
Penalised MR-Egger & $0.83(0.51-1.34)$ & 0.452 & 0.657 \\
Simple median & $0.80(0.49-1.30)$ & 0.375 & $\mathrm{NA}$ \\
Weighted median & $0.84(0.62-1.15)$ & 0.278 & $\mathrm{NA}$ \\
Penalised weighted median & $0.84(0.62-1.15)$ & 0.278 & $\mathrm{NA}$ \\
\hline
\end{tabular}

${ }^{a}$ Change in colorectal cancer risk per unit log-transformed 25-hydroxyvitamin $\mathrm{D}$ (nmol/L)

$C l$ confidence interval, $I V W$ inverse variance weighted, MR Mendelian randomisation, $N A$ not available, $P_{\text {int }} P$ value of Egger regression test on the intercept

designing and investing in future trials on the effects of vitamin $\mathrm{D}$ in high-risk population subgroups.

Our previous MR study did not detect a statistically significant causal effect of 25-OHD on CRC [15]. Another recent MR study with 11,488 CRC cases did not show a causal relationship between circulating vitamin D level and CRC risk [17]. However, the conclusions might have been limited by lower statistical power. Insufficient power has been a major shortcoming of MR studies, because genetic variants usually explain only a very small proportion of the exposure variation on the liability scale. Those four variants could only explain $3.6 \%$ to $5.2 \%[53,54]$ of variance on $25-\mathrm{OHD}$, thus leading to potentially low statistical power. Our previous study included 2001 CRC cases and 2237 controls, but only reached a power of 0.35 to detect $25 \%$ decreased risk per standard deviation increase in 25-OHD [15]. We recently reported the largest ever GWAS on circulating 25-OHD concentrations in which we identified two additional genetic loci contributing to the genetic architecture of 25-OHD [20]. Using these six variants, we developed a stronger instrument compared with the previous four-variant instrument (F-statistic 46.0 vs. 13.0 in SOCCS controls) [15]. However, the overall heritability calculated using linkage disequilibrium score regression analysis [20] was modest, with $2.84 \%$ out of $7.5 \%$ overall heritability explained by the identified GWAS variants. Although the addition of new GWAS variants provided only limited improvement in the strength of the IV, overall statistical power was substantially improved in our current 25-OHD-CRC MR analysis. With data from the largest GWAS studies on 25-OHD and $\mathrm{CRC}$, as well as more individual CRC cases involved in this MR study, we have a power of 0.80 at the $\alpha$ level of 0.05 to identify a $19 \%$ decreased CRC risk per standard deviation increase in 25-OHD for the individual level approach using $9940 \mathrm{CRC}$ cases and 22,848 controls from the UK biobank CRC case-control dataset, Croatia and Scottish CRC case-control series, and a power of 0.80 to identify a $14.3 \%$ decreased CRC risk for the summary level approaches using 17,716 cases and 40,095 controls across 11 individual GWASs.

The validity of MR estimates of causal effects requires that several assumptions be held. First, for the relevance assumption, we only included the strongest independent variants identified by the largest GWAS so they were all robustly associated with the exposure. Second, none of the genetic variants used in our analysis were cited by the NHGRI-EBI Catalogue of published GWAS as associated with known CRC risk confounders (such as height, BMI, alcohol consumption, smoking, type II diabetes, inflammatory bowel disease, adenomas) [55]. Furthermore, our genetic instrument was not associated with age, sex, BMI, smoking status, alcohol consumption, physical activity and assessment centre, suggesting no effects of violated IV second assumption due to tested confounders on final study conclusion. However, we cannot rule out the possibility of association between our IV and an unknown and/or unmeasured confounding factor. Finally, to assess violations of the exclusion restriction assumption or 'no pleiotropy', we employed a range of methods known to robustly account for horizontal pleiotropy, including MR-Egger and a weighted median approach. All of the methods showed similar results and MR-Egger intercept indicated no evidence of pleiotropic effects, suggesting robust null findings.

Our study had sufficient power and an appropriate design to formally address the hypothesis of a causal relationship between low circulating vitamin D and CRC risk. We also used a range of various MR approaches. Another strength of our study was the availability of collected information on known confounding factors such as height, weight, BMI, age and sex, which allowed testing the MR assumptions of independent associations between IV and confounders. However, there were some limitations too. Firstly, due to the low proportion of $25-\mathrm{OHD}$ variance $(2.84 \%)$ explained by the genetic variants and relatively small sample size, our individual level data analysis did not reach the desired power $(<0.80)$ assuming true causal effects of $25 \mathrm{OHD}$ on CRC risk was similar to the effect observed in the observational SOCCS case-control study (OR 0.83). The study had sufficient power to identify a causal effect larger than 21.7\% decreased CRC risk per 25-OHD standard deviation. Although the summary statistics approach included a larger sample size, we only had a power of 0.49 if the true causal effect was less than 10\% decreased CRC risk per 25-OHD standard deviation. Similarly, both approaches were underpowered if the real proportion of 
$25-\mathrm{OHD}$ variance explained by the IV was $2 \%$ and below. Secondly, for individual level analysis, circulating 25-OHD levels from the SOCCS dataset were measured in the Scottish population, which manifested a significantly lower average level compared with other European populations [8]; this could possibly weaken the strength of our genetic instrument. A weak IV is an issue for the summary two-step MR approach too. The MR estimates are known to be biased towards the null in the presence of a weak IV (F statistics < 5) [56, 57]. This is similar to regression dilution bias in an observational study due to non-differential measurement errors. However, given the strength of the IV (F-statistic 46.0) used in the present analysis and the large sample size in the summary level approach, the bias towards the null is unlikely to affect our results. We also cannot exclude the possibility of collider bias due to the non-representative selection of participants into the study cohorts. Selection bias is present to some degree in all epidemiological studies. Evidence of a 'healthy volunteer' selection bias has been described for the UK biobank $[58,59]$. The collider bias can lead to an association between the IV and the outcome in the absence of a causal effect as well as to underestimation of real causal effects in some cases [60]. It seems, though, that in most cases collider bias effect is smaller than pleiotropy or population stratification bias [60]. Finally, as in many previous MR studies, the current paper is based on the assumption of a linear effect between CRC risk and 25-OHD levels. Indeed, two recent studies on CRC have shown a linear relationship between 25-OHD and CRC $[61,62]$. In particular, results from a recent dose-response meta-analysis of observational studies [61] as well as the analysis of the EPIC study [62] support a linear relationship between 25-OHD and CRC. Nevertheless, it is still possible that the assumption of linearity may not hold true. There are some recently suggested IV methods that can test non-linear exposure-outcome effects, but the methods are not fully developed yet [63, 64]. Furthermore, these approaches require access to individual level data, which is a limiting factor for many MR studies including ours. Finally, although application of a linear IV in the case of a non-linear relationship between the exposure and outcome could not give any insight into the shape of the relationship, it is still possible to provide population-averaged causal effects [64].

\section{Conclusions}

In conclusion, this MR study provides further evidence that genetically determined lower circulating levels of 25-OHD are unlikely to have a causal effect on CRC risk with strength on the order of the effects previously reported in observational studies. Observed associations may be due to confounders and reverse causation, although a very small causal effect of 25-OHD on CRC risk cannot be ruled out. Future research might be best focused on understanding the mechanisms of the relationship between CRC and circulating 25-OHD.

\section{Additional file}

Additional file 1: Study description, imputation and genetic analysis and supplementary Tables S1-S6. (DOC $211 \mathrm{~kb}$ )

\section{Abbreviations}

25-OHD: 25-hydroxyvitamin D; BMI: body mass index; Cl: confidence interval; CRC: colorectal cancer; GRS: genetic risk scores; GWAS: genome-wide association study; IV: instrumental variable; IVW: inverse variance weighted; LBC: Lothian Birth Cohort; MR: Mendelian randomisation; OR: odds ratio; SOCCS: Study of Colorectal Cancer in Scotland

\section{Acknowledgements}

We are gratefull to the SUNLIGHT consortium for sharing summary data and Xia Jiang, Elina Hyppönen, Peter Kraft and Douglas P. Kiel on behalf of the SUNLIGHT consortium.

\section{Funding}

We acknowledge support from program grant no. C348/A18927 from Cancer Research UK. The work was also supported by a project grant (to MGD) within the MRC Human Genetics Unit Centre Grant (U127527202 and U127527198 from 1/4/18). YH, XL and XM are supported by the China Scholarship Council. ET is supported by a CRUK Career Development Fellowship (grant no.C31250/A22804). IJD and SEH are supported by the University of Edinburgh Centre for Cognitive Ageing and Cognitive Epidemiology, which is funded by the Medical Research Council and the Biotechnology and Biological Sciences Research Council (grant no. MR/K026992/1). The Lothian Birth Cohort studies are funded by Age UK (Disconnected Mind project) and the Biotechnology and Biological Sciences Research Council (grant no. BB/F019394/1). Genotyping of the GS:SFHS samples was carried out by the Edinburgh Clinical Research Facility, University of Edinburgh, and was funded by the Medical Research Council UK and the Wellcome Trust (Wellcome Trust Strategic Award 'STratifying Resilience and Depression Longitudinally' (STRADL), Reference 104036/Z/14/Z). GS:SFHS received core support from the Scottish Executive Health Department, Chief Scientist Office, grant number CZD/16/6. The MRC provides core funding to the QTL in Health and Disease research program at the MRC HGU, IGMM, University of Edinburgh.

\section{Availability of data and materials}

Details of genotyping and quality control of UK biobank available at: http:// biobank.ndph.ox.ac.uk/crystal/docs/genotyping_qc.pdf

Details of genotype imputation of UK biobank available at: http:// www.ukbiobank.ac.uk/wp-content/uploads/2014/04/ imputation_documentation_May2015.pdf

Participants description of UK biobank available at: https://www.biorxiv.org/ content/biorxiv/early/2017/07/20/166298.full.pdf

\section{Authors' contributions}

ET and MGD conceived the study. YH, XL, XM, AS and MT performed data analysis and MT had overall supervisory responsibility for data analysis. $\mathrm{YH}$ and MT drafted the manuscript with ET and MGD. HC, SMF, PVS and LZ revised the manuscript. PVS and VS collected data for the SOCCS study. IJD and SEH provided data from the Lothian Birth Cohort studies. $\mathrm{CH}, \mathrm{AC}$ and DP provided data from the GS:SFHS studies. KV, IK and MF provided data from the Croatian cohort. SMF, MW, ET and MGD generated CRC case and control vitamin D data. XJ, EH, PK and DPK led the recently published GWAS meta-analysis in the SUNLIGHT Consortium. RH, IPT and MGD led the GWAS meta-analysis on CRC. All authors critically reviewed the manuscript and contributed important intellectual content. All authors have read and approved the final manuscript as submitted.

\section{Ethics approval and consent to participate}

SOCCS received ethical and management approvals from the MultiCentre Research Ethics committee for Scotland (approval number MREC/ 01/0/5), 18 
Local Research Ethics committees, 18 Caldicott guardians and 16 NHS Trust management committees.

GS:SFHS: 05/S1401/89 Tayside Committee on Medical Research Ethics A, Generic Research Tissue Bank approval: GS:SFHS: 10/S1402/20 Tayside Committee on Medical Research Ethics B.

Ethics permission for the Lothian Birth Cohort 1921 (LBC1921) was obtained from the Lothian Research Ethics Committee (LREC/1998/4/183).

Ethics permission for the Lothian Birth Cohort 1936 (LBC1936) was obtained from the Multi-Centre Research Ethics Committee for Scotland (MREC/01/0/56) and the Lothian Research Ethics Committee (LREC/2003/2/29).

The research activities of UK Biobank were approved by the North West Multi-centre Research Ethics Committee (11/NW/0382) in relation to the process of participant invitation, assessment and follow-up procedures. Additionally, ethics approvals from the National Information Governance Board for Health \& Social Care in England and Wales and approval from the Community Health Index Advisory Group in Scotland were also obtained to gain access to the information that would allow the invitation of participants. This study did not need to recontact the participants, and no separate ethics approval was required according to the Ethics and Governance Framework (EGF) of UK Biobank. The approved data request application ID for this analysis is 7441 .

\section{Consent for publication}

No consent for publication was required.

\section{Competing interests}

The authors declare that they have no competing interests.

\section{Publisher's Note}

Springer Nature remains neutral with regard to jurisdictional claims in published maps and institutional affiliations.

\section{Author details}

'Colon Cancer Genetics Group, Medical Research Council Human Genetics Unit, Medical Research Council Institute of Genetics \& Molecular Medicine, Western General Hospital, The University of Edinburgh, Edinburgh EH4 2XU, UK. ${ }^{2}$ West China School of Medicine/West China Hospital, Sichuan University, Chengdu 610041, People's Republic of China. ${ }^{3}$ Centre for Global Health Research, Usher Institute of Population Health Sciences and Informatics, The University of Edinburgh, Edinburgh EH8 9AG, UK. ${ }^{4}$ Department of Public Health and Primary Care, Institute of Population Health, Trinity College Dublin, University of Dublin, Dublin 24, D02 PN40, Ireland. ${ }^{5}$ Program in Genetic Epidemiology and Statistical Genetics. Department of Epidemiology, Harvard T.H.Chan School of Public Health, 677 Huntington Avenue, Boston, MA 02115, USA. ${ }^{6}$ Unit of Cardiovascular Epidemiology, Institute of Environmental Medicine, Karolinska Institutet, Nobels vagen 13, Stockholm 17177, Sweden. ${ }^{7}$ Australian Centre for Precision Health, University of South Australia Cancer Research Institute, University of South Australia, Adelaide, SA 5001, Australia. ${ }^{8}$ Population, Policy and Practice, University College London, Great Ormond Street, Institute of Child Health, WC1E 6BT, London, UK. ${ }^{9}$ Institute for Aging Research, Hebrew SeniorLife, 1200 Centre Street, Boston, MA 02131, USA. ${ }^{10}$ Department of Medicine, Beth Israel Deaconess Medical Center and Harvard Medical School, Boston, MA 02115, USA. "'Broad Institute of Harvard and Massachusetts Institute of Technology, Boston, MA 02142, USA. ${ }^{12}$ MRC Human Genetics Unit, MRC Institute of Genetics \& Molecular Medicine, The University of Edinburgh, Western General Hospital, Edinburgh EH4 2XU, UK. ${ }^{13}$ Generation Scotland, Institute of Genetics and Molecular Medicine, The University of Edinburgh, Western General Hospital Edinburgh, Crewe Road, Edinburgh EH4 2XU, UK. ${ }^{14}$ Agency for Medicinal Products and Medical Devices, Department for Quality, Safety and Efficacy Assessment, Zagreb, Croatia. ${ }^{15}$ Department of Surgical Oncology, University Hospital for Tumours, Sestre milosrdnice University Hospital Centre, Zagreb, Croatia. ${ }^{16}$ School of Medicine, University of Zagreb, Zagreb, Croatia. ${ }^{17}$ Centre for Cognitive Ageing and Cognitive Epidemiology, The University of Edinburgh, Edinburgh, UK. ${ }^{18}$ Centre for Genomic and Experimental Medicine, Institute of Genetics and Molecular Medicine, The University of Edinburgh, Edinburgh, UK. ${ }^{19}$ Department of Psychology, The University of Edinburgh, Edinburgh, UK. ${ }^{20}$ Division of Genetics and Epidemiology, The Institute of Cancer Research, Sutton, Surrey SM2 5NG, UK. ${ }^{21}$ Institute of Cancer and Genomic Sciences, University of Birmingham, Birmingham, UK.
Received: 17 April 2018 Accepted: 6 July 2018

Published online: 14 August 2018

\section{References}

1. Torre LA, Bray F, Siegel RL, Ferlay J, Lortet-Tieulent J, Jemal A. Global cancer statistics, 2012. CA Cancer J Clin. 2015;65:87-108.

2. Haggar FA, Boushey RP. Colorectal cancer epidemiology: incidence, mortality, survival, and risk factors. Clin Colon Rectal Surg. 2009;22:191-7.

3. Cross HS. Vitamin D: synthesis and catabolism- considerations for Cancer causation and therapy. In: Trump DL, Johnson CS, editors. Vitamin D and Cancer. New York: Springer New York; 2011. p. 1-24.

4. Feldman D, Krishnan AV, Swami S, Giovannucci E, Feldman BJ. The role of vitamin $\mathrm{D}$ in reducing cancer risk and progression. Nat Rev Cancer. 2014; 14:342-57.

5. Ma Y, Zhang P, Wang F, Yang J, Liu Z, Qin H. Association between vitamin $D$ and risk of colorectal cancer: a systematic review of prospective studies. J Clin Oncol. 2011;29:3775-82.

6. Theodoratou E, Tzoulaki I, Zgaga L, loannidis JP. Vitamin D and multiple health outcomes: umbrella review of systematic reviews and meta-analyses of observational studies and randomised trials. BMJ. 2014;348:92035.

7. Hossein-nezhad A, Holick MF. Vitamin D for health: a global perspective. Mayo Clin Proc. 2013;88:720-55.

8. Zgaga L, Theodoratou E, Farrington SM, Agakov F, Tenesa A, Walker M, et al. Diet, environmental factors, and lifestyle underlie the high prevalence of vitamin D deficiency in healthy adults in Scotland, and supplementation reduces the proportion that are severely deficient. J Nutr. 2011;141:1535-42.

9. Vimaleswaran KS, Berry DJ, Lu C, Tikkanen E, Pilz S, Hiraki LT, et al. Causal relationship between obesity and vitamin $\mathrm{D}$ status: bi-directional Mendelian randomization analysis of multiple cohorts. PLoS Med. 2013;10:e1001383.

10. Giovannucci E. Epidemiology of vitamin D and colorectal cancer. Anti Cancer Agents Med Chem. 2013;13:11-9.

11. Manson JE, Bassuk SS, Lee IM, Cook NR, Albert MA, Gordon D, et al. The VITamin D and OmegA-3 TriaL (VITAL): rationale and design of a large randomized controlled trial of vitamin $D$ and marine omega-3 fatty acid supplements for the primary prevention of cancer and cardiovascular disease. Contemp Clin Trials. 2012;33:159-71.

12. Didelez V, Sheehan N. Mendelian randomization as an instrumental variable approach to causal inference. Stat Methods Med Res. 2007;16:309-30.

13. Gupta V, Walia GK, Sachdeva MP. 'Mendelian randomization': an approach for exploring causal relations in epidemiology. Public Health. 2017;145:113-9.

14. Zheng J, Baird D, Borges MC, Bowden J, Hemani G, Haycock P, et al. Recent developments in Mendelian randomization studies. Curr Epidemiol Rep. 2017:4:330-45.

15. Theodoratou E, Palmer T, Zgaga L, Farrington SM, McKeigue P, Din FV, et al. Instrumental variable estimation of the causal effect of plasma 25-hydroxyvitamin D on colorectal cancer risk: a mendelian randomization analysis. PLoS One. 2012;7:e37662.

16. Hiraki LT, Qu C, Hutter CM, Baron JA, Berndt SI, Bezieau S, et al. Genetic predictors of circulating 25 -hydroxyvitamin d and risk of colorectal cancer. Cancer Epidemiol Biomarkers Prev. 2013;22:2037-46.

17. Dimitrakopoulou VI, Tsilidis KK, Haycock PC, Dimou NL, Al-Dabhani K, Martin RM, et al. Circulating vitamin D concentration and risk of seven cancers: Mendelian randomisation study. BMJ. 2017;359:j4761.

18. Zgaga L, Agakov F, Theodoratou E, Farrington SM, Tenesa A, Dunlop $M G$, et al. Model selection approach suggests causal association between 25-hydroxyvitamin D and colorectal cancer. PLoS One. 2013;8: e63475.

19. Wang $T J$, Zhang F, Richards JB, Kestenbaum B, van Meurs JB, Berry D, et al. Common genetic determinants of vitamin $D$ insufficiency: a genome-wide association study. Lancet. 2010;376:180-8.

20. Jiang X, O'Reilly PF, Aschard H, Hsu YH, Richards JB, Dupuis J, et al. Genome-wide association study in 79,366 European-ancestry individuals informs the genetic architecture of 25-hydroxyvitamin D levels. Nat Commun. 2018;9:260.

21. Pierce $B L$, Ahsan $H$, Vanderweele TJ. Power and instrument strength requirements for Mendelian randomization studies using multiple genetic variants. Int J Epidemiol. 2011;40:740-52.

22. Palmer TM, Lawlor DA, Harbord RM, Sheehan NA, Tobias JH, Timpson NJ, et al. Using multiple genetic variants as instrumental variables for modifiable risk factors. Stat Methods Med Res. 2012;21:223-42. 
23. Study C, Houlston RS, Webb E, Broderick P, Pittman AM, Di Bernardo MC, et al. Meta-analysis of genome-wide association data identifies four new susceptibility loci for colorectal cancer. Nat Genet. 2008;40:1426-35.

24. Houlston RS, Cheadle J, Dobbins SE, Tenesa A, Jones AM, Howarth K, et al. Meta-analysis of three genome-wide association studies identifies susceptibility loci for colorectal cancer at 1q41, 3q26.2, 12q13.13 and 20q13.33. Nat Genet. 2010;42:973-7.

25. Theodoratou E, Farrington SM, Tenesa A, McNeill G, Cetnarskyj R, Barnetson RA, et al. Dietary vitamin B6 intake and the risk of colorectal cancer. Cancer Epidemiol Biomarkers Prev. 2008;17:171-82.

26. Deary IJ, Gow AJ, Pattie A, Starr JM. Cohort profile: the Lothian birth cohorts of 1921 and 1936. Int J Epidemiol. 2012;41:1576-84.

27. Smith BH, Campbell A, Linksted P, Fitzpatrick B, Jackson C, Kerr SM, et al. Cohort profile: generation Scotland: Scottish family health study (GS:SFHS). The study, its participants and their potential for genetic research on health and illness. Int J Epidemiol. 2013;42:689-700.

28. Timofeeva MN, Kinnersley B, Farrington SM, Whiffin N, Palles C, Svinti V, et al. Recurrent coding sequence variation explains only a small fraction of the genetic architecture of colorectal Cancer. Sci Rep. 2015;5:16286.

29. Allen N, Sudlow C, Downey P, Peakman T, Danesh J, Elliott P, et al. UK biobank: current status and what it means for epidemiology. Health Policy Technol. 2012;1:123-6.

30. Orlando G, Law PJ, Palin K, Tuupanen S, Gylfe A, Hanninen UA, et al. Variation at 2q35 (PNKD and TMBIM1) influences colorectal cancer risk and identifies a pleiotropic effect with inflammatory bowel disease. Hum Mol Genet. 2016;25:2349-59.

31. Rodriguez-Broadbent H, Law PJ, Sud A, Palin K, Tuupanen S, Gylfe A, et al. Mendelian randomisation implicates hyperlipidaemia as a risk factor for colorectal cancer. Int J Cancer. 2017;140:2701-8.

32. Wald $A$. The fitting of straight lines if both variables are subject to error. Ann Math Stat. 1940;11:284-300.

33. Lawlor DA, Harbord RM, Sterne JA, Timpson N, Davey Smith G. Mendelian randomization: using genes as instruments for making causal inferences in epidemiology. Stat Med. 2008;27:1133-63.

34. Burgess S, Thompson SG. Use of allele scores as instrumental variables for Mendelian randomization. Int J Epidemiol. 2013:42:1134-44.

35. Houlston RS, Members of COGENT. COGENT (COlorectal cancer GENeTics) revisited. Mutagenesis. 2012:27:143-51.

36. Whiffin N, Hosking FJ, Farrington SM, Palles C, Dobbins SE, Zgaga L, et al. Identification of susceptibility loci for colorectal cancer in a genome-wide meta-analysis. Hum Mol Genet. 2014;23:4729-37.

37. Al-Tassan NA, Whiffin N, Hosking FJ, Palles C, Farrington SM, Dobbins SE, et al. A new GWAS and meta-analysis with 1000Genomes imputation identifies novel risk variants for colorectal cancer. Sci Rep. 2015;5:10442.

38. Burgess S, Scott RA, Timpson NJ, Davey Smith G, Thompson SG, Consortium E-l. Using published data in Mendelian randomization: a blueprint for efficient identification of causal risk factors. Eur J Epidemiol. 2015;30: 543-52.

39. Bowden J, Davey Smith G, Haycock PC, Burgess S. Consistent estimation in Mendelian randomization with some invalid instruments using a weighted median estimator. Genet Epidemiol. 2016;40:304-14.

40. Bowden J, Davey Smith G, Burgess S. Mendelian randomization with invalid instruments: effect estimation and bias detection through egger regression. Int J Epidemiol. 2015;44:512-25.

41. Burgess S, Bowden J, Dudbridge F, Thompson SG. Robust instrumental variable methods using multiple candidate instruments with application to Mendelian randomization. arXiv preprint. 2016;arXiv:160603729. https://arxiv. org/abs/1606.03729. Accessed 23 July 2018.

42. Brion MJ, Shakhbazov K, Visscher PM. Calculating statistical power in Mendelian randomization studies. Int J Epidemiol. 2013:42:1497-501.

43. Yavorska OO, Burgess S. Mendelian randomization: an R package for performing Mendelian randomization analyses using summarized data. Int J Epidemiol. 2017;46(6):1734-9.

44. Wactawski-Wende J, Kotchen JM, Anderson GL, Assaf AR, Brunner RL, O'Sullivan MJ, et al. Calcium plus vitamin D supplementation and the risk of colorectal cancer. N Engl J Med. 2006;354:684-96.

45. Baron JA, Barry EL, Mott LA, Rees JR, Sandler RS, Snover DC, et al. A trial of calcium and vitamin D for the prevention of colorectal adenomas. N Engl J Med. 2015;373:1519-30.

46. Forman MR, Levin B. Calcium plus vitamin D3 supplementation and colorectal cancer in women. N Engl J Med. 2006;354:752-4.
47. Newmark HL, Wargovich MJ, Bruce WR. Colon cancer and dietary fat, phosphate, and calcium: a hypothesis. J Natl Cancer Inst. 1984;72:1323-5.

48. Zhang $X$, Giovannucci E. Calcium and vitamin D for the prevention of colorectal adenomas. N Engl J Med. 2016;374:791.

49. Barry EL, Peacock JL, Rees JR, Bostick RM, Robertson DJ, Bresalier RS, et al. Vitamin D receptor genotype, vitamin D3 supplementation, and risk of colorectal adenomas: a randomized clinical trial. JAMA Oncol. 2017;3:628-35.

50. Meeker S, Seamons A, Paik J, Treuting PM, Brabb T, Grady WM, et al. Increased dietary vitamin D suppresses MAPK signaling, colitis, and colon cancer. Cancer Res. 2014;74:4398-408.

51. Newmark HL, Yang K, Kurihara N, Fan K, Augenlicht LH, Lipkin M. Westernstyle diet-induced colonic tumors and their modulation by calcium and vitamin D in C57BI/6 mice: a preclinical model for human sporadic colon cancer. Carcinogenesis. 2009;30:88-92.

52. Irving AA, Plum LA, Blaser WJ, Ford MR, Weng C, Clipson L, et al. Cholecalciferol or 25-hydroxycholecalciferol neither prevents nor treats adenomas in a rat model of familial colon cancer. J Nutr. 2015;145:291-8.

53. Ye Z, Sharp SJ, Burgess S, Scott RA, Imamura F, InterAct Consortium, et al. Association between circulating 25-hydroxyvitamin D and incident type 2 diabetes: a mendelian randomisation study. Lancet Diabetes Endocrinol. 2015:3:35-42.

54. Hiraki LT, Major JM, Chen C, Cornelis MC, Hunter DJ, Rimm EB, et al. Exploring the genetic architecture of circulating 25-hydroxyvitamin D. Genet Epidemiol. 2013;37:92-8.

55. MacArthur J, Bowler E, Cerezo M, Gil L, Hall P, Hastings E, et al. The new NHGRI-EBI catalog of published genome-wide association studies (GWAS catalog). Nucleic Acids Res. 2017:45:D896-901.

56. Pierce BL, Burgess S. Efficient design for Mendelian randomization studies: subsample and 2-sample instrumental variable estimators. Am J Epidemiol. 2013;178:1177-84

57. Burgess S, Thompson SG. Bias in causal estimates from Mendelian randomization studies with weak instruments. Stat Med. 2011;30:1312-23.

58. Munafo MR, Tilling K, Taylor AE, Evans DM, Davey Smith G. Collider scope: when selection bias can substantially influence observed associations. Int J Epidemiol. 2018;47:226-35.

59. Ganna A, Ingelsson E. 5 year mortality predictors in 498,103 UK biobank participants: a prospective population-based study. Lancet. 2015;386:533-40.

60. Apostolos G, Burgess S. Contextualizing selection bias in Mendelian randomization: how bad is it likely to be? arXiv preprint 2018;arXiv:1803. 03987. https://arxiv.org/abs/1803.03987. Accessed 23 July 2018.

61. Garland CF, Gorham ED. Dose-response of serum 25-hydroxyvitamin D in association with risk of colorectal cancer: a meta-analysis. J Steroid Biochem Mol Biol. 2017;168:1-8.

62. Jenab M, Bueno-de-Mesquita $H B$, Ferrari $P$, van Duijnhoven FJ, Norat $T$, Pischon $T$, et al. Association between pre-diagnostic circulating vitamin D concentration and risk of colorectal cancer in European populations:a nested case-control study. BMJ. 2010;340:b5500.

63. Silverwood RJ, Holmes MV, Dale CE, Lawlor DA, Whittaker JC, Smith GD et al. Testing for non-linear causal effects using a binary genotype in a Mendelian randomization study: application to alcohol and cardiovascular traits. Int J Epidemiol. 2014;43:1781-90.

64. Burgess S, Davies NM, Thompson SG, EPIC-InterAct Consortium. Instrumental variable analysis with a nonlinear exposure-outcome relationship. Epidemiology. 2014;25:877-85.

65. Timpson NJ, Wade $\mathrm{KH}$, Smith GD. Mendelian randomization: application to cardiovascular disease. Curr Hypertens Rep. 2012;14:29-37.

\section{Ready to submit your research? Choose BMC and benefit from}

- fast, convenient online submission

- thorough peer review by experienced researchers in your field

- rapid publication on acceptance

- support for research data, including large and complex data types

- gold Open Access which fosters wider collaboration and increased citations

- maximum visibility for your research: over $100 \mathrm{M}$ website views per year

At $\mathrm{BMC}$, research is always in progress.

Learn more biomedcentral.com/submission 